\title{
Leveraging information in parking assistance
}

\section{systems}

\author{
Evangelia Kokolaki Merkourios Karaliopoulos and Ioannis Stavrakakis \\ Department of Informatics and Telecommunications \\ National \& Kapodistrian University of Athens \\ Ilissia, 15784 Athens, Greece \\ Email: \{evako, mkaralio, ioannis\}@di.uoa.gr
}

\begin{abstract}
This paper seeks to systematically explore the efficiency of uncoordinated parking space allocation in urban environments with two types of parking facilities. Drivers decide whether to go for inexpensive but limited on-street parking spots or expensive yet over-dimensioned parking lots, incurring an additional cruising cost when deciding for on-street parking spots but failing to actually acquire one. Their decisions are made under perfect knowledge of the total parking supply and cost, and different levels of information about the parking demand. In general, this information can be broadcast by a centralized parking assistance system or collected and shared opportunistically among vehicles.

We investigate the impact of information and pricing on the parking space allocation process by taking a game-theoretic approach. The uncoordinated parking spot selection problem is formulated as an instance of resource selection games and drivers are viewed as strategic decision-makers that aim at minimizing the cost of the acquired parking spot. Three different game variants capture the drivers' interactions when deciding under complete/probabilistic information and uncertainty, respectively, about the parking demand. We analyze them, derive their equilibria, and compute the related Price of Anarchy values by comparing the induced social cost at these equilibria against the optimal one, i.e., the cost under an ideal centralized parking spot reservation system.

It is shown that, for typical prices of the two types of parking facilities, drivers tend to over-compete for the on-street parking space giving rise to redundant cruising cost. Yet this inefficiency can be alleviated through appropriate choice of parking costs and the placement of parking lots but also through the systematic manipulation of the information that is announced to the drivers. Counterintuitive less-ismore effects emerge regarding the way information availability modulates the resulting social cost, which underpin general competitive service provision settings. Our results and discussion provide useful hints
\end{abstract}


for the practical management of parking facilities and contribute to the better understanding of effective information dissemination mechanisms in emerging smart city environments.

\section{INTRODUCTION}

The tremendous increase of urbanization necessitates the efficient and environmentally sustainable management of various urban processes and operations. Recent advances in wireless networking and sensing technologies can address this need by enabling efficient monitoring mechanisms for these processes and higher flexibility to control them, thus paving the way for the so-called smart cities. Intelligent networked sensor nodes (i.e., smartdust), placed on buildings' surfaces or mounted on vehicles, constitute pervasive monitoring platforms that can measure environmental parameters such as pollution concentration, radiation level, road traffic congestion or public transport utilization. The data generated by this monitoring infrastructure can then be processed by municipal authorities to more efficiently manage both the environmental and man-made city resources. In the case of parking space resources, in particular, the challenge is to efficiently manage the available parking space and reduce the vehicle volumes that cruise in search of it, in order to alleviate not only traffic congestion but also the related environmental burden.

To this end, academic research but also public and/or private initiatives in the past years have been primarily directed towards the design and deployment of two mechanisms. The first one is summarized under the term parking assistance systems. Common to these systems is the exploitation of wireless communications and information sensing technologies to collect and broadcast (in centralized mechanisms, i.e., in [1], [2]) or share (in distributed systems, i.e., in [3], [4]) information about the supply of (and demand for) parking space resources. This information ideally saves drivers from redundant cruising trips in search of a parking spot and assists in the management of parking resources, with centralized systems even implementing parking spot reservation. The second mechanism is pricing. The application

of pricing schemes on the parking facilities aims to improve parking availability in overused parking zones and reduce double-parking and excessive cruising phenomena (i.e., in [5]).

This paper seeks to systematically explore the impact of these two mechanisms on the efficiency of the parking search process and the parking resource utilization. In particular, we consider scenarios where the parking resource allocation is not controlled by a centralized entity, e.g., through a reservation mechanism. The drivers choose independently to either compete for the inexpensive but scarce on-street parking spots or head for the more expensive parking lot(s). In the first case, they run the risk of failing to get a spot and having to a posteriori take the more expensive alternative, this time suffering the additional cruising cost in terms of time, fuel consumption (and stress) of the failured attempt. Drivers make their decisions 
drawing on various levels of information about the parking demand (number of drivers) and perfect knowledge of the parking capacity (supply) and the applied pricing schemes on the parking facilities. The questions that arise in this respect are: How do different amounts of information on the parking demand modulate drivers' parking choices? Could this be controlled by the parking service operator to minimize the cost that drivers incur and the redundant cruising cost? How do prices charged for the two types of parking facilities modulate the information impact?

We take a game-theoretic approach and view the drivers as rational and strategic selfish agents that try to minimize the cost the actual humans/drivers pay for the acquired parking space. In fact, we consider automatic software agent implementations rather than human decision-makers yet, the actual human/driver undertakes the action with the assumption that she fully complies with the machines' suggestions. We formulate the uncoordinated parking spot selection problem as an instance of resource selection games in Section II. The accuracy of the information that drivers possess about the parking demand is abstracted at three levels giving rise to an equal number of game variants. In Section III, we describe the game variant under complete information, which establishes a first comparison reference for the efficiency of the uncoordinated parking spot selection process. Whereas in Section IV we analyze two more realistic scenarios, where drivers make their decisions under probabilistic information and full uncertainty, respectively. First, we derive the drivers' parking choices under the stable operational states in which all competing influences are balanced, that is, the equilibria of all three game variants. Then we quantify the efficiency of the uncoordinated parking search process by computing the related Price of Anarchy values, which compare the induced social cost at these equilibria against the optimal one, i.e., the cost under an ideal centralized system possessing perfect information about the status of parking spots, reserving spare ones for the drivers, and directing them thereto. Ultimately, we explore the factors that affect the Price of Anarchy results and hence, can modulate the efficiency of the parking spot selection.

The numerical results in Section V suggest that, for typical prices of the two types of parking resources, drivers tend to over-compete for the on-street parking spots giving rise to redundant cruising cost. However, there are important degrees of freedom in alleviating this inefficiency. One possibility is through the choice of charging costs for the two facilities. A second, much less intuitive, possibility is through the systematic manipulation of information that becomes available to the drivers. In this respect, counterintuitive less-is-more effects emerge, implying that the possession of less accurate information on the parking demand alleviates competition and induces equilibria behaviors that outperform those under complete knowledge.

We outline related research and position our work against it in Section VI and conclude with the 
discussion in Section VII that identifies other instances of networking problems lending to a formulation as resource selection games; draws parallels with other mechanisms, where the information becomes an asset shaping nodes' behavior; and, finally, motivates further research on the game-theoretic assertions regarding the drivers' behavior, taking as a starting point insights from the cognitive psychology domain.

\section{MOdELING THE PARKING SPOT SELECTION PROCESS}

The traffic and environmental burden of the parking space search process depends on several factors. The potential overlap in drivers' travel destinations, personal parking preferences, and the drivers' unwillingness to park beyond the proximity of their destination have been identified as causes for major congestion problems. These are further aggravated due to specific parking regulations-restrictions, which are often in place especially in center areas of big cities, e.g., business districts. Typically, parking in these areas is completely forbidden or restricted in whole sets of road blocks so that the effective curbside is scarce (see Fig. 1). Thus, drivers may have to settle for parking spots that hardly satisfy personal search criteria (i.e., short walking distance to their destinations). More fundamentally, drivers are faced with a decision whether to compete for the low-cost but scarce on-street parking space or directly head for the over-dimensioned but more expensive parking lots.

In our model, drivers that manage to park in curbside pay $c_{o s p, s}$ per-time cost units, whereas those heading directly for the safer parking lot option pay $c_{p l}=\beta \cdot c_{o s p, s}, \beta>1$, per-time cost units. However, drivers that decide first to search for low-cost parking spots but fail to acquire one and finally resort to a parking lot, pay $c_{o s p, f}=\gamma \cdot c_{o s p, s}, \gamma>\beta$, per-time cost units. The excess cost $\delta \cdot c_{o s p, s}$, with $\delta=\gamma-\beta>0$, reflects the actual cost of cruising and the "virtual" cost of wasted time till eventually reaching the more expensive parking facility. Parking facilities of both types are managed by a single operator, e.g., municipal authorities, and all parking facilities of the same type are assumed to be of similar value to the drivers -we discuss this assumption further in Section VII. Thus, the drivers' decisions are essentially made on the two sets of parking facilities, i.e., on-street parking space vs. parking lots, rather than individual set items, i.e., parking spots.

Optimal centralized parking spot allocation: Under the optimal centralized parking spot allocation scheme, the full information processing and decision-making tasks lie with a central entity. Drivers issue their parking requests to a central server, which monitors the parking space, possesses precise information about its availability, and assigns it so that the overall cost paid by drivers is minimized (typically, the case when the municipality runs centralized parking assistance services). Thus, in an urban environment with $R$ on-street parking spots, whereby such an ideal centralized system serves the parking requests of 

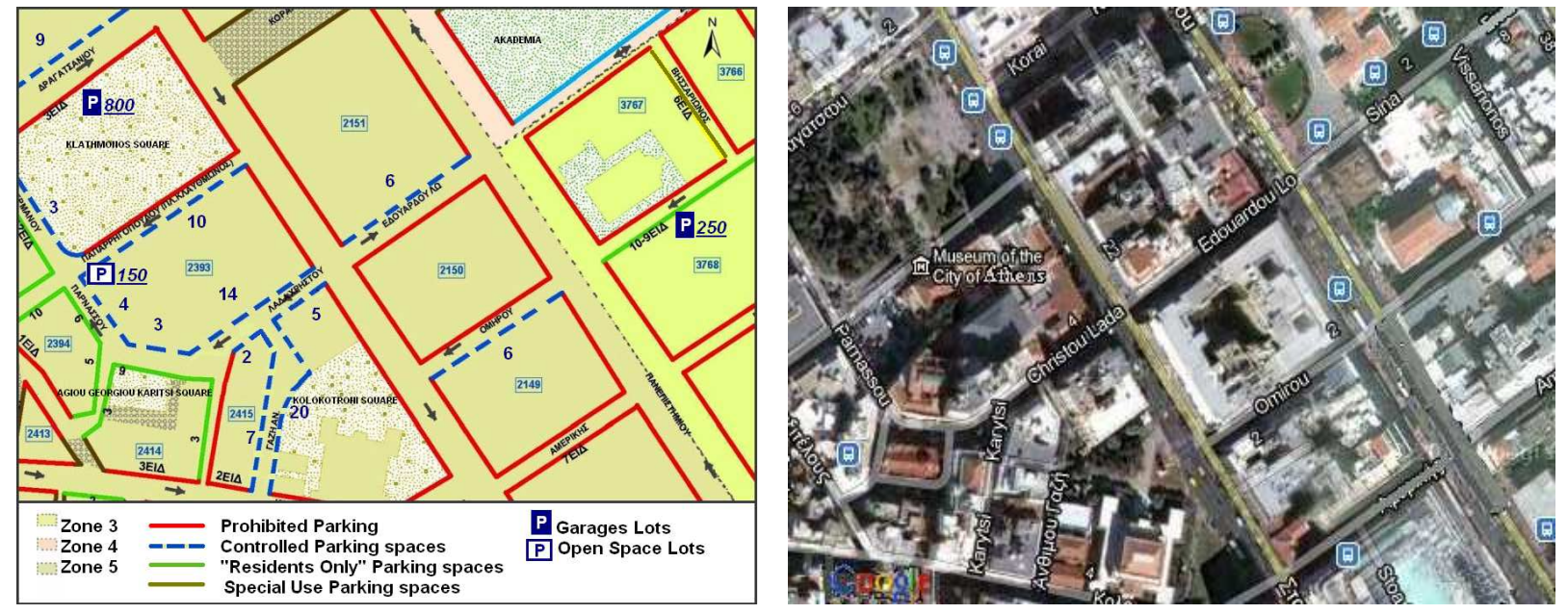

Fig. 1. Parking and geographical map (thanks to Google maps) of the center area of Athens, Greece. Dashed lines show metered controlled (on-street) parking spots, whereas "P" denotes parking lots. The map illustrates, as well, the capacity of both parking options.

$N(\geq R)$ drivers, exactly $R(N-R)$ drivers would be directed to the low-cost (resp. more expensive) facilities and no one would pay the excess cruising cost.

Uncoordinated parking spot selection: In the absence of central coordination, each driver acts selfishly aiming at minimizing what she will pay for a parking spot. However, the intuitive tendency to head for the low-cost on-street parking space, combined with its scarcity in urban center areas, give rise to tragedy of commons effects [6] and highlight the game-theoretic dynamics behind the parking spot selection task. Thus, the collective decision-making on parking space selection can be formulated as an instance of resource selection games, whereby $N$ players (i.e., drivers) compete against each other for a finite number $R$ of common resources (i.e., curbside parking) [7]. In this game-theoretic view of the parking spot selection process, the drivers are assumed to be rational strategic players. They explicitly consider the presence of identical counter-actors that also make rational decisions, weight the costs related to every possible action profile, and act as cost-minimizers. In doing so, they may or may not hold precise information about the actual demand, i.e., competition, for parking resources.

In the following sections, we consider the uncoordinated drivers' behavior within a particular time window over which they reach a city center. In general, these synchronization phenomena in drivers' flow occur at specific time zones during the day [8]. We analyze the parking spot selection game under different levels of uncertainty (or amounts of information) for the overall parking demand, ranging from exact (complete) knowledge to simply an upper bound on the potential competitors. In all cases, we derive the stable operational conditions and the associated costs incurred by the players, and compare them with those under optimal centralized parking spot allocation. 


\section{PARKING SPOT SELECTION UNDER COMPLETE KNOWLEDGE OF PARKING DEMAND}

Ideally, the players (i.e., drivers) determine their strategy under complete knowledge of those parameters that shape their cost. Given the symmetry of the game, the additional piece of information that is considered available to the players, besides the number of vacant parking spots and the employed pricing scheme, is the level of parking demand, i.e., the number of drivers searching for parking space. More formally, the one-shot ${ }^{1}$ parking spot selection game under complete knowledge of the parking demand is defined as follows:

Definition 3.1: A strategic Parking Spot Selection Game is a tuple $\Gamma(N)=\left(\mathcal{N}, \mathcal{R},\left(A_{i}\right)_{i \in \mathcal{N}}\right.$, $\left.\left(w_{j}\right)_{j \in(o s p, p l)}\right)$, where:

- $\mathcal{N}=\{1, \ldots, N\}, N>1$ is the set of drivers who seek for parking space,

- $\mathcal{R}=\mathcal{R}_{o s p} \cup \mathcal{R}_{p l}$ is the set of parking spots; $\mathcal{R}_{o s p}$ is the set of on-street spots, with $R=\left|\mathcal{R}_{\text {osp }}\right| \geq 1$; $\mathcal{R}_{p l}$ the set of spots in parking lot, with $\left|\mathcal{R}_{p l}\right| \geq N$,

- $A_{i}=\{o s p, p l\}$ is the action set for each driver $i \in \mathcal{N}$, comprising of the actions "on-street" (osp) and "parking lot" (pl),

- $w_{o s p}(\cdot)$ and $w_{p l}(\cdot)$ are the cost functions of the two actions, respectively ${ }^{2}$.

The parking spot selection game falls under the broader family of congestion games $^{3}$. The players' payoffs (here: costs) are non-decreasing functions of the number of players competing for the parking capacity, rather than their identities, and common to all players. More specifically, drivers who decide to compete for the curbside parking space undergo the risk of not being among the $R$ winner-drivers to get a spot. In this case, they have to eventually resort to a parking lot, only after wasting extra time and fuel (plus patience supply) on the failed attempt. The expected cost for a driver that plays the action $o s p$, $w_{\text {osp }}: A_{1} \times \ldots \times A_{N} \rightarrow \mathbb{R}$, is therefore a function of the number of drivers $k$ taking it, and is given by

$$
w_{o s p}(k)=\min (1, R / k) c_{o s p, s}+(1-\min (1, R / k)) c_{o s p, f}
$$

On the other hand, the cost for those that head directly to the parking lot facilities is fixed

$$
w_{p l}(k)=c_{p l}=\beta \cdot c_{o s p, s}
$$

\footnotetext{
${ }^{1}$ The study of the dynamic variant of the game is a natural direction for future work.

${ }^{2}$ Note that the cost functions are defined over the action set of each user; in the original definition of resource selection games in [7], cost functions are defined over the resources but the resource set coincides with the action set.

${ }^{3}$ Readers who are more familiar with game theory will notice a resemblance to the atomic variant of Pigou's selfish routing example [9]. Pigou's paths correspond to the two parking alternatives, one having a high user-independent use cost and the other a cost that scales with the number of users (albeit not linearly).
} 

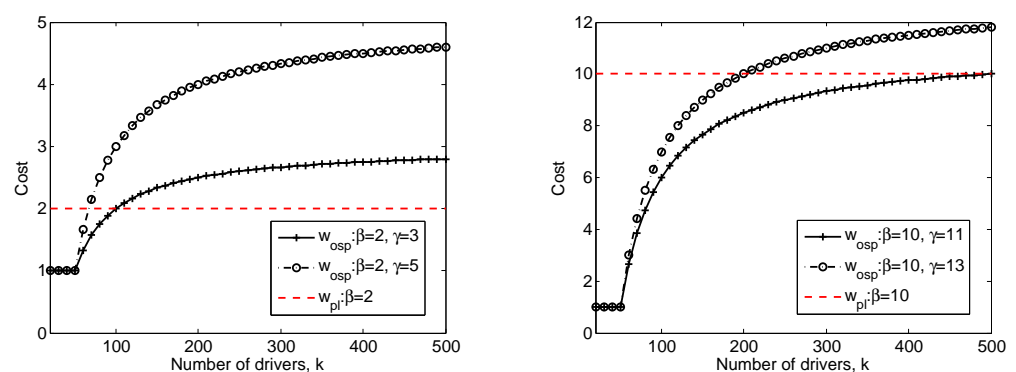

Fig. 2. The cost functions for parking spots in curbside and in parking lots: $R=50, c_{o s p, s}=1$.

Figure 2 plots the cost functions against the number of drivers, for both parking options, under different pricing schemes.

We denote an action profile by the vector $a=\left(a_{i}, a_{-i}\right) \in \times{ }_{k=1}^{N} A_{k}$, where $a_{-i}$ denotes the actions of all other drivers but player $i$ in the profile $a$. Besides the two pure strategies reflecting the pursuit of parking spots in curbside and in parking lots, the drivers may also randomize over them. In particular, if $\Delta\left(A_{i}\right)$ is the set of probability distributions over the action set of player $i$, a player's mixed action corresponds to a vector $p=\left(p_{\text {osp }}, p_{p l}\right) \in \Delta\left(A_{i}\right)$, where $p_{\text {osp }}$ and $p_{p l}$ are the probabilities of the pure actions "on-street" and "parking lot", respectively, with $p_{o s p}+p_{p l}=1$, while its cost is a weighted sum of the cost functions $w_{o s p}(\cdot)$ and $w_{p l}(\cdot)$ of the pure actions. We draw on concepts from [10] and theoretical results from [7], [11] to derive the equilibrium strategies for the game $\Gamma(N)$ and assess their (in)efficiency.

\section{A. Pure equilibrium strategies}

Existence: The parking spot selection game constitutes a symmetric game, where the action set is common to all players and consists of two possible actions, "on-street" and "parking lot". Cheng et al. have shown in ([11], Theorem 1) that every symmetric game with two strategies has an equilibrium in pure strategies.

Derivation: Due to the game's symmetry, the full set of $2^{N}$ different action profiles maps into $N+1$ different action meta-profiles. Each meta-profile $a(m), m \in[0, N]$ encompasses all $\left(\begin{array}{l}N \\ m\end{array}\right)$ different action profiles that correspond to the same number of drivers competing for on-street parking space. The expected costs for these $m$ drivers and for the $N-m$ ones choosing directly the parking lot alternative are functions of $a(m)$ rather than the exact action profile.

In general, the cost $c_{i}^{N}\left(a_{i}, a_{-i}\right)$ for driver $i$ under the action profile $a=\left(a_{i}, a_{-i}\right)$ is 


$$
c_{i}^{N}\left(a_{i}, a_{-i}\right)= \begin{cases}w_{o s p}\left(N_{o s p}(a)\right), & \text { for } a_{i}=o s p \\ w_{p l}\left(N-N_{o s p}(a)\right), & \text { for } a_{i}=p l\end{cases}
$$

where $N_{o s p}(a)$ is the number of competing drivers for on-street parking under action profile $a$. Equilibria action profiles combine the players' best-responses to their opponents' actions. Formally, an action profile $a=\left(a_{i}, a_{-i}\right)$ is a pure Nash equilibrium if for all $i \in \mathcal{N}$, it holds that $a_{i} \in$ $\arg \min _{a_{i}^{\prime} \in A_{i}}\left(c_{i}^{N}\left(a_{i}^{\prime}, a_{-i}\right)\right)$, so that no player has anything to gain by changing her decision unilaterally.

Therefore, to derive the equilibria states, we determine the conditions on $N_{o s p}$ that break the equilibrium definition and reverse them. More specifically, given an action profile $a$ with $N_{o s p}(a)$ competing drivers, a player gains by changing her decision to play action $a_{i}$ in two circumstances:

$$
\begin{aligned}
& \text { when } a_{i}=p l \text { and } w_{o s p}\left(N_{o s p}(a)+1\right)<c_{p l} \\
& \text { when } a_{i}=o s p \text { and } w_{o s p}\left(N_{o s p}(a)\right)>c_{p l}
\end{aligned}
$$

Taking into account the relation between the number of drivers and the available on-street parking spots, $R$, we can postulate the following Lemma:

Lemma 3.1: In the strategic parking spot selection game $\Gamma(N)$, a driver is motivated to change his action $a_{i}$ in the following circumstances:

- $a_{i}=p l$ and (a) $N_{o s p}(a)<R \leq N$ or (b) $R \leq N_{o s p}(a)<N_{0}-1 \leq N$ or (c) $N_{o s p}(a)<N \leq R$

- $a_{i}=o s p$ and $R<N_{0}<N_{o s p}(a) \leq N$

where $N_{0}=\frac{R(\gamma-1)}{\delta} \in \mathbb{R}$.

Proof: Conditions (6a) and (6c) are trivial. Since the current number of competing vehicles is less than the on-street parking capacity, every driver having originally chosen the parking lot option has the incentive to change her decision due to the price differential between $c_{o s p, s}$ and $c_{p l}$.

When $N_{o s p}(a)$ exceeds the curbside parking supply, a driver who has decided to avoid competition, profits from switching her action when (4) holds, which combined with (1), yields (6b). Similarly, a driver that first decides to compete, profits by switching her action if (5) holds, which combined with (1), yields (7).

The following Theorem for the pure Nash equilibria of the parking spot selection game may now be stated.

Theorem 3.1: The strategic parking spot selection game $\Gamma(N)$ has:

(a) for $N \leq N_{0}$, a unique Nash equilibrium profile $a^{*}$ with $N_{o s p}\left(a^{*}\right)=N_{o s p}^{N E, 1}=N$

(b.1) for $N>N_{0}$ and $N_{0} \in(R, N) \backslash \mathbb{N}^{*},\left(\begin{array}{c}N \\ \left\lfloor N_{0}\right\rfloor\end{array}\right)$ Nash equilibrium profiles $a^{\prime}$ with $N_{o s p}\left(a^{\prime}\right)=$ 


$$
N_{o s p}^{N E, 2}=\left\lfloor N_{0}\right\rfloor
$$

(b.2) for $N>N_{0}$ and $N_{0} \in[R+1, N] \cap \mathbb{N}^{*},\left(\begin{array}{c}N \\ N_{0}\end{array}\right)$ Nash equilibrium profiles $a^{\prime}$ with $N_{o s p}\left(a^{\prime}\right)=$ $N_{o s p}^{N E, 2}=N_{0}$ and $\left(\begin{array}{c}N \\ N_{0}-1\end{array}\right)$ Nash equilibrium profiles $a^{\star}$ with $N_{o s p}\left(a^{\star}\right)=N_{o s p}^{N E, 3}=N_{0}-1$.

Proof: Theorem 3.1 follows directly from Lemma 3.1. The equilibrium states satisfy both the conditions $N_{o s p} \geq \frac{R(\gamma-1)}{\delta}-1$ and $N_{o s p} \leq \frac{R(\gamma-1)}{\delta}$. Thus, the game has two equilibrium states on $N_{o s p}$ for $N>N_{0}$ with integer $N_{0}$ (case b.2), or a unique state, otherwise (cases a, b.1).

In Appendix A, we provide an alternative way to derive the equilibria of $\Gamma(N)$ via potential functions.

Efficiency: The efficiency of the equilibria action profiles resulting from the strategically selfish decisions of the drivers is assessed through the broadly used metric of the Price of Anarchy [10]. It expresses the ratio of the social cost in the worst-case equilibria over the optimal social cost under ideal coordination of the drivers' strategies.

Proposition 3.1: In the parking spot selection game, the pure Price of Anarchy equals:

$$
\mathrm{P} \circ \mathrm{A}=\left\{\begin{array}{l}
\frac{\gamma N-(\gamma-1) \min (N, R)}{\min (N, R)+\beta \max (0, N-R)}, \quad \text { if } N_{0} \geq N \\
\frac{\left\lfloor N_{0}\right\rfloor \delta-R(\gamma-1)+\beta N}{R+\beta(N-R)}, \quad \text { if } N_{0}<N
\end{array}\right.
$$

Proof: The social cost under action profile $a$ equals:

$$
C\left(N_{o s p}(a)\right)=\sum_{i=1}^{N} c_{i}^{N}(a)=\left\{\begin{array}{l}
c_{o s p, s}\left(N \beta-N_{o s p}(a)(\beta-1)\right), \text { if } N_{o s p}(a) \leq R \\
c_{o s p, s}\left(N_{o s p}(a) \delta-R(\gamma-1)+\beta N\right), \text { if } R<N_{o s p}(a) \leq N
\end{array}\right.
$$

The numerators of the two ratios are obtained directly by replacing the first two $N_{o s p}^{N E}$ values (a) and (b1) (worst-cases) computed in Theorem 3.1. On the other hand, under the socially optimal action profile $a_{\text {opt }}$, exactly $R$ drivers pursue on-street parking space, whereas the remaining $N-R$ go directly to a parking lot. Therefore, under $a_{o p t}$, no drivers find themselves in the unfortunate situation to have to pay the additional cost of cruising in terms of time and fuel after having unsuccessfully competed for the on-street parking spots. The optimal social cost, $C_{o p t}$ is given by:

$$
C_{\text {opt }}=\sum_{i=1}^{N} c_{i}^{N}\left(a_{\text {opt }}\right)=c_{o s p, s}[\min (N, R)+\beta \cdot \max (0, N-R)]
$$

Proposition 3.2: In the parking spot selection game, the pure Price of Anarchy is upper-bounded by $\frac{1}{1-R / N}$ with $N>R$.

Proof: The condition is obtained directly from Proposition 3.1, when $N>R$. 


\section{B. Mixed-action equilibrium strategies}

We mainly draw our attention on symmetric mixed-action equilibria since these can be more helpful in dictating practical strategies in real systems. Asymmetric mixed-action equilibria are discussed at the end of the section.

Existence: In ([7], Theorem 1) it is proved that a unique symmetric mixed equilibrium exists for the broader family of resource selection games with more than two players and increasing cost functions. This is easily shown to hold for the parking spot selection game $\Gamma(N)$, with $N>R$ and cost functions $w_{o s p}(\cdot)$ and $w_{p l}(\cdot)$ that are non-decreasing functions of the number of players (increasing and constant, respectively).

Derivation: Let $p=\left(p_{o s p}, p_{p l}\right)$ denote a mixed-action. Then, the expected costs of choosing parking spots in curbside and in parking lot, when all other drivers play the mixed-action $p$, are given by

$$
\begin{aligned}
c_{i}^{N}(o s p, p) & =\sum_{N_{o s p}=0}^{N-1} w_{o s p}\left(N_{o s p}+1\right) B\left(N_{o s p} ; N-1, p_{o s p}\right) \\
c_{i}^{N}(p l, p) & =c_{p l}
\end{aligned}
$$

where $B\left(N_{o s p} ; N-1, p_{\text {osp }}\right)$ is the Binomial probability distribution with parameters $N-1$ and $p_{\text {osp }}$, for $N_{o s p}$ drivers choosing curbside parking. The cost of the symmetric profile where everyone plays the mixed-action $p$ is given by

$$
c_{i}^{N}(p, p)=p_{\text {osp }} \cdot c_{i}^{N}(\text { osp }, p)+p_{p l} \cdot c_{i}^{N}(p l, p)
$$

With these at hand, we can now postulate the following Theorem.

Theorem 3.2: The strategic parking spot selection game $\Gamma(N)$ has a unique symmetric mixed-action Nash equilibrium $p^{N E}=\left(p_{o s p}^{N E}, p_{p l}^{N E}\right)$, where $p_{o s p}^{N E}=1$, if $N \leq N_{0}$ and $p_{o s p}^{N E}=\frac{N_{0}}{N}$, if $N>N_{0}$, with $p_{o s p}^{N E}+p_{p l}^{N E}=1$ and $N_{0} \in \mathbb{R}$.

Proof: The proof is given in Appendix B.

Asymmetric mixed-action equilibria: In the analysis of pure equilibria in Section III-A, we showed that there may exist multiple asymmetric pure equilibria, when the number of drivers exceeds $N_{0}$. In general, the derivation of results for asymmetric mixed-action equilibria is much harder than for either their pure or their symmetric counterparts since the search space is much larger. Moreover, asymmetric mixed-equilibria have two more undesirable properties: a) they do not treat all players equally, i.e., different players end up with a-priori worse chances to come up with an inexpensive parking spot; b) their realization in practical situations is much more difficult than that of their symmetric counterparts. Therefore, we focus our analysis and discussion on symmetric equilibria and their (in)efficiency. 


\section{PARKING SPOT SELECTION UNDER INCOMPLETE KNOWLEDGE OF PARKING DEMAND}

The availability of complete information about the drivers' (i.e., players') population is a fairly strong and unrealistic assumption. In this section we relax it and study two game variants under incomplete demand information, where the players either share common probabilistic information about the overall parking demand or are totally uncertain about it. Note that the parking service operator, depending on the network and information sensing infrastructure at her disposal, may provide the competing drivers with different amounts of information about the demand for parking space (e.g., based on historical statistical data). However, again, drivers are assumed to have perfect knowledge of the parking supply; this is fairly realistic and feasible since the sensing of the parking space and the broadcasting of the collected data can be viewed as less complex tasks.

\section{A. Probabilistic knowledge of parking demand}

In the Bayesian model of the game, the drivers determine their actions on the basis of private information, their types. The type in this game is a binary variable indicating whether a driver is in search of parking space (active player) or not. Every driver knows her own type along with the strategy space, the cost functions, and the possible types of all others. However, she ignores the real state of the game at a particular moment in time, as expressed by the types of the other players, and, hence, she cannot deterministically reason out the actions being played. Instead, she draws on common prior probabilistic information about the activity of drivers to derive estimates about the expected cost of her actions. Formally, the Bayesian parking spot selection game is defined as follows:

Definition 4.1: A Bayesian Parking Spot Selection Game is a tuple $\Gamma_{B}(N)=\left(\mathcal{N}, \mathcal{R},\left(A_{i}\right)_{i \in \mathcal{N}}\right.$,

$\left.\left(w_{j}\right)_{j \in(\text { osp }, p l)},\left(\Theta_{i}\right)_{i \in \mathcal{N}}, f_{\Theta}\right)$, where $\mathcal{N}$ and $\mathcal{R}$, are the driver and parking spot set, as defined for $\Gamma(N)$, and

- $A_{i}=\{o s p, p l, \oslash\}$, the set of potential actions for each driver $i \in \mathcal{N}$,

- $\Theta_{i}=\{0,1\}$, the set of types for each driver $i \in \mathcal{N}$, where 1 (0) stands for active (inactive) drivers, - $S_{i}: \Theta_{i} \rightarrow A_{i}$, the set of possible strategies for each driver $i \in \mathcal{N}$,

- $c_{i}^{N_{B}}(s(\vartheta), \vartheta)$, the cost functions for each driver $i \in \mathcal{N}$, for every type profile $\vartheta \in \times_{k=1}^{N} \Theta_{k}$ and strategy profile $s(\vartheta) \in \times_{k=1}^{N} S_{k}$, that are functions of $w_{o s p}(\cdot)$ and $w_{p l}(\cdot)$ as defined for $\Gamma(N)$, and also written as $c_{i}^{N_{B}}(s(\vartheta), \vartheta)=c_{i}^{N_{B}}\left(s_{i}\left(\vartheta_{i}\right), s_{-i}\left(\vartheta_{-i}\right), \vartheta_{i}, \vartheta_{-i}\right)$,

- $p_{a c t}$ is the probability for a driver to be active, i.e., interested in available parking space.

In $\Gamma_{B}(N)$, all inactive drivers $i$ abstain from the game; hence, $s_{i}\left(\vartheta_{i}=0\right)=\oslash$. On the contrary, for active players $i, s_{i}\left(\vartheta_{i}=1\right) \in\{o s p, p l\}$, under pure-action strategy, or $s_{i}\left(\vartheta_{i}=1\right) \in \Delta(\{o s p, p l\})$, when 
they randomize over this subset of $A_{i}$ under mixed-action strategy. The game is symmetric when, besides the action set, drivers share the same activity probability, $p_{a c t}$ and hence, the same prior joint probability distribution of the drivers' activity (types), $f_{\Theta}$. The number of active players upon each time depends on their types and is given by $n_{a c t}=\sum_{k} \vartheta_{k}$. The action profile is the effect of players' strategies on their types and is noted as $a=(s(\vartheta), \vartheta) \in \times{ }_{k=1}^{N} A_{k}$.

Equilibria: For the Bayesian parking spot selection game, the strategy profile $s^{\prime} \in \times_{k=1}^{N} S_{k}\left(\vartheta_{k}=1\right)$ is a Bayesian Nash equilibrium if for all $i \in \mathcal{N}$ with $\vartheta_{i}=1$ :

$$
\begin{gathered}
s_{i}\left(\vartheta_{i}\right) \in \arg \min _{s_{i}^{\prime} \in S_{i}}\left(c_{i}^{N_{B}}\left(s_{i}\left(\vartheta_{i}\right), s_{-i}\left(\vartheta_{-i}\right), \vartheta_{i}, \vartheta_{-i}\right)\right) \quad \text { or, } \\
s_{i}\left(\vartheta_{i}\right) \in \arg \min _{s_{i}^{\prime} \in S_{i}} \sum_{\vartheta_{-i}} f_{\Theta}\left(\vartheta_{-i} / \vartheta_{i}\right) c_{i}^{\sum_{k} \vartheta_{k}}\left(s_{i}^{\prime}, s_{-i}\left(\vartheta_{-i}\right), \vartheta_{i}, \vartheta_{-i}\right)
\end{gathered}
$$

where $c_{i}^{\sum_{k} \vartheta_{k}}\left(s_{i}^{\prime}, s_{-i}\left(\vartheta_{-i}\right), \vartheta_{i}, \vartheta_{-i}\right)$, with $s_{l}\left(\vartheta_{l}=0\right)=p l, \forall l \neq i$, is the cost $c_{i}^{m}\left(s_{i}^{\prime}, s_{-i}\right)$ of driver $i$ under profile $s$ in the game $\Gamma(m)$ with $m=\sum_{k} \vartheta_{k}$ drivers, and $f_{\Theta}\left(\vartheta_{-i} / \vartheta_{i}\right)$ the posterior conditional probability of the active drivers given that user $i$ is active, as derived from the application of the Bayesian rule. Therefore, $s^{\prime}$ minimizes the expected cost over all possible combinations of the other drivers' types and strategies so that no active player can further lower its expected cost by unilaterally changing her strategy.

Theorem 4.1: The Bayesian parking spot selection game $\Gamma_{B}(N)$ has unique symmetric equilibrium profiles $p^{N E_{B}}=\left(p_{o s p}^{N E_{B}}, p_{p l}^{N E_{B}}\right)$, with $p_{o s p}^{N E_{B}}+p_{p l}^{N E_{B}}=1$. More specifically:

- a unique pure (Bayesian Nash) equilibrium with $p_{o s p}^{N E_{B}}=1$, if $p_{a c t}<\frac{N_{0}}{N}$,

- a unique symmetric mixed-action Bayesian Nash equilibrium with $p_{o s p}^{N E_{B}}=\frac{N_{0}}{N p_{a c t}}$, if $p_{a c t} \geq \min \left(\frac{N_{0}}{N}, 1\right)$, where $N_{0} \in \mathbb{R}$.

Proof: We present the proof in Appendix C.

\section{B. Strictly incomplete information about parking demand}

The worst-case scenario with respect to the information drivers possess for making their decisions is represented by the pre-Bayesian game variant. In the pre-Bayesian parking spot selection game, the drivers may hold some knowledge about the upper limit of the vehicles that are potential competitors for parking resources, yet their actual number is not known, not even probabilistically.

Pre-Bayesian games do not necessarily have ex-post Nash equilibria, even in mixed actions. The expost Nash equilibrium consists of strategies that, for every joint type profile, result in actions which are in Nash equilibrium in the corresponding strategic game. On the other hand, all quasi-concave pre-Bayesian 
TABLE I

EQUILIBRIUM STRATEGIES FOR THE STRATEGIC, BAYESIAN AND PRE-BAYESIAN PARKING SPOT SELECTION GAME

\begin{tabular}{|c|c|c|}
\hline \multicolumn{3}{|c|}{ strategic Parking Spot Selection Game, $\Gamma(N)$} \\
\hline \hline Condition & Equilibrium type & Equilibrium expression \\
\hline$N \leq N_{0}, N_{0} \in \mathbb{R}$ & pure Nash Eq & $N_{o s p}^{N E}=N$ \\
$N>N_{0}, N_{0} \in(R, N) \backslash \mathbb{N}^{*}$ & pure Nash Eq & $N_{o s p}^{N E}=\left\lfloor N_{0}\right\rfloor$ \\
$N>N_{0}, N_{0} \in[R+1, N] \cap \mathbb{N}^{*}$ & pure Nash Eq & $N_{o s p}^{N E}=N_{0}, N_{o s p}^{N E}=N_{0}-1$ \\
$N>N_{0}, N_{0} \in \mathbb{R}$ & mixed-action Nash Eq & $p_{\text {osp }}^{N E}=\frac{N_{0}}{N}$ \\
\hline \hline \multicolumn{2}{|c|}{ Bayesian Parking Spot Selection Game, $\Gamma_{B}(N)$} \\
\hline \hline \multicolumn{2}{|c|}{ pondition } & Equilibrium type \\
\hline$p_{a c t}<\frac{N_{0}}{N}, N_{0} \in \mathbb{R}$ & pure Bayesian Nash Eq & $p_{o s p}^{N E_{B}}=1$ \\
$p_{a c t} \geq \min \left(\frac{N_{0}}{N}, 1\right), N_{0} \in \mathbb{R}$ & mixed-action Bayesian Nash Eq & $p_{o s p}^{N E_{B}}=\frac{N_{0}}{N p_{a c t}}$ \\
\hline \hline \multicolumn{2}{|c|}{ pre-Bayesian Parking Spot Selection Game, $\Gamma_{p B}(N)$} \\
\hline \hline Condition & Equilibrium type & Equilibrium expression \\
\hline$N \leq N_{0}, N_{0} \in \mathbb{R}$ & pure safety-level Eq & $p_{o s p}^{N E_{p B}}=1$ \\
$N>N_{0}, N_{0} \in \mathbb{R}$ & mixed-action safety-level Eq & $p_{o s p}^{N E_{p B}}=\frac{N_{0}}{N}$ \\
\hline
\end{tabular}

games do have at least one mixed-strategy safety-level equilibrium [7]. In the safety-level equilibrium, every player minimizes over her strategy set $S_{i}$ the worst-case (maximum) cost she may suffer over all possible types and actions of her competitors $\left(S_{-i}, \Theta_{-i}\right)$.

The result of interest for our pre-Bayesian variant of the parking spot selection model $\Gamma_{p B}(N)$ is the following Proposition, due to [7], whose implications for the efficiency of the equilibria behaviors of the drivers are discussed in Section V.

Proposition 4.1: An action profile $a$ is the unique symmetric mixed-action safety-level equilibrium of the pre-Bayesian parking spot selection game, $\Gamma_{p B}(N)$, with non-decreasing resource cost functions, iff $a$ is the unique symmetric mixed-action equilibrium of the respective strategic game with deterministic knowledge of the number of players, $\Gamma(N)$.

In Table I we summarize the equilibrium strategies for the three variants of the parking spot selection game.

\section{NUMERICAL RESUlTS}

The analysis in Sections III and IV suggests that four important factors affect the (in)efficiency of the parking search process. The first two are the parking demand and the supply of parking facilities, encompassing on-street parking space supply but also the number and location of parking lots that 


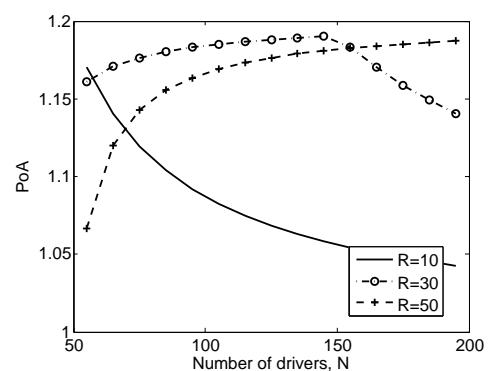

a. $2 D \operatorname{POA}(N)$

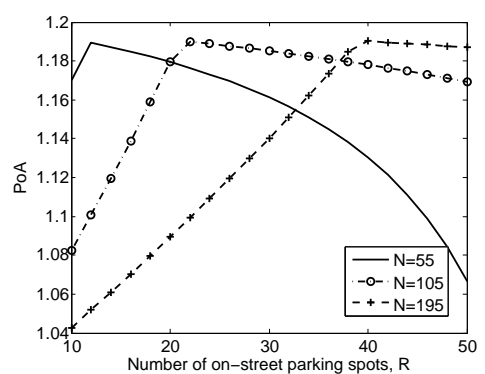

b. $2 D \mathrm{POA}(R)$

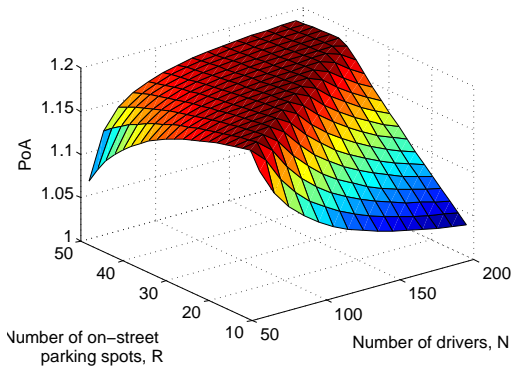

c. $3 D \operatorname{POA}(N, R)$

Fig. 3. Price of Anarchy as a function of the parking demand and supply, under fixed pricing scheme $\beta=5, \delta=1$.

indirectly determine the drivers' cruising cost. The third factor is the pricing scheme, i.e., the charging costs set for on-street parking and parking lots, while the less intuitive fourth one is the information available to the drivers when taking their decisions.

When parking resources are managed by a single operator, e.g., a municipality, all factors but the parking demand are under its control. Hence, in the long-term, the operator copes with the parking demand through strategic planning of the parking space supply, involving re-dimensioning the available on-street parking space and constructing(relocating) new(existing) parking lots. Whereas, in the shortterm, it can do so by manipulating the prices charged for the two types of parking facilities. The fourth factor has implications for both short- and long-term management processes. If mechanisms that collect and disseminate information about parking demand (e.g., local radio channel transmitting statistical data about parking space utilization) are already in place, then the operator can directly control the information announced to the drivers; if not, it may decide to make an investment and deploy them since, as shown later in this section, this provides it with an additional degree of freedom in shaping the behaviors of the drivers and the efficiency of the uncoordinated parking search process.

In the remainder of the section, we draw on $P O A$ results to quantify this inefficiency. We first outline its dependence on the parking demand and supply; then we discuss in more detail the impact of the two factors that lend to shorter-term manipulation, pricing and information availability. For the numerical results we adopt per-time unit normalized cost values used in typical municipal parking systems in big European cities [8]. The parking fee for on-street space is set to $c_{o s p, s}=1$ unit whereas the cost of parking lots $\beta$ ranges in $(1,16]$ units; the excess cruising cost parameter $\delta$ is let vary within $[1,5]$ units. 


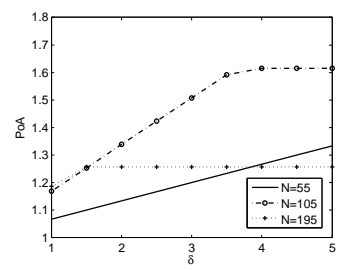

a. $2 D \operatorname{POA}(N, \delta), R=50$

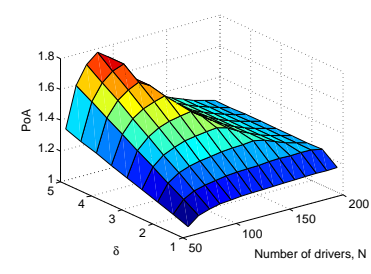

b. $3 D \operatorname{POA}(N, \delta), R=50$

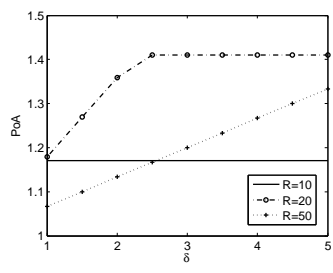

c. $2 D \mathrm{POA}(R, \delta), N=55$

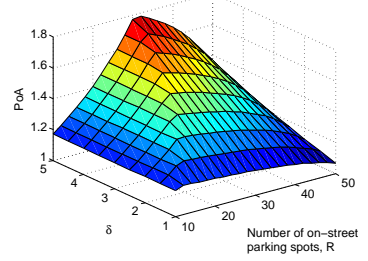

d. $3 D \mathrm{POA}(R, \delta), N=55$

Fig. 4. Price of Anarchy as a function of the parking demand and supply, under variable cruising cost $\delta$ and fixed pricing scheme $\beta=5$.

\section{A. Impact of parking demand and on-street parking supply}

An optimal centralized mechanism would assign exactly $\min (N, R)$ on-street parking spots to $\min (N, R)$ drivers. If $N \leq R$, in the absence of central coordination, all drivers pay the low fee for on-street parking space and, trivially, $\mathrm{P} \circ \mathrm{A}=1$. Hereafter, we focus on the more interesting case $N>R$, where a number of drivers end up paying the extra cruising cost $\delta c_{o s p, s}$ (see Lemma 3.1, Theorem 3.1). Under a fixed pricing scheme, this inefficiency depends on the curbside parking supply as well as the intensity of the parking requests. In Figure 3, we plot the POA against $N$ and $R$ ranging in $[55,195]$ and $[10,50]$, respectively. The following remarks suggest joint conditions on $N$ and $R$ that result in more efficient parking search, namely:

Varying $N$ : For $N \leq N_{0}$ it holds that $\frac{\vartheta P o A}{\vartheta N}>0$ and therefore, the P०A is strictly increasing in $N$. On the contrary, for $N>N_{0}$, the $\mathrm{P} \circ \mathrm{A}$ is strictly decreasing in $N$, since $\frac{\vartheta P o A}{\vartheta N}<0$. Figure 3 a illustrates these trends for three different values of $R$. The P०A is shown to take its maximum(worst) value at $N=N_{0}$, if $N_{0} \in[55,195]$. Thus, for $R=30$, the worst P०A value occurs at $N=150$, whereas the other two curves appear either strictly decreasing $\left(N_{0}=50\right.$, for $\left.R=10\right)$ or strictly increasing $\left(N_{0}=250\right.$, for $R=50$ ) for all considered $N$ values.

Varying $R$ : For $N \leq N_{0}$ or, equivalently, for $R \geq \frac{N \delta}{\gamma-1}$, it holds that $\frac{\vartheta P o A}{\vartheta R}<0$. Therefore, the PoA is strictly decreasing in $R$. For $R<\frac{N \delta}{\gamma-1}$, we get $\frac{\vartheta P o A}{\vartheta R}>0$ and hence, the $\mathrm{P} \circ \mathrm{A}$ is strictly increasing in $R$. In Figure 3b, we plot the P०A against $R$ for three different $N$ values. In all three cases, the highest inefficiency appears at the corresponding $R=\frac{N \delta}{\gamma-1}$ value.

When all drivers choose to compete, that is, if $N \leq N_{0}$ or $R \geq \frac{N \delta}{\gamma-1}$, exactly $R$ drivers pay $c_{o s p, s}$ while the rest of them, i.e., $N-R$ drivers, pay $\gamma c_{o s p, s}$. Thus, under a fixed pricing scheme, the social cost is optimized when the maximum charging cost $\left(\gamma c_{o s p, s}\right)$ is incurred by the minimum possible set of drivers, namely when the parking demand is the lowest possible one $(N=R+1)$ or, equivalently, as the curbside parking capacity is increased so that only one driver fails the competition for the low-cost 
parking spots.

On the other hand, when $N>N_{0}$ or $R<\frac{N \delta}{\gamma-1}, R$ drivers pay $c_{o s p, s}, N-N_{0}$ drivers pay $\beta c_{o s p, s}$ and $N_{0}-R$ drivers pay $\gamma c_{o s p, s}$. Under the optimal operation of the service, the latter two sets of drivers head directly for space in parking lot. Thus, the efficiency of the uncoordinated parking search is improved as the parking demand increases, making the total cost paid by the $N-N_{0}$ drivers the most critical factor for the overall social cost and hence, minimizing the total cost paid by the $N_{0}-R$ drivers due to the lack of coordination. In another approach, the set of $N_{0}-R=\frac{R(\beta-1)}{\delta}$ drivers that fail the competition, is minimized when the on-street parking capacity becomes the lowest possible one, i.e., $R=1$.

When the on-street parking capacity cannot be re-dimensioned, the parking service operator typically resorts to the construction of new parking lots to alleviate the parking search problem. This policy might change the extra cruising cost $\delta$. Figure 4 displays the $\mathrm{P} \cap \mathrm{A}$ for $\delta$ ranging in $[1,5]$ and suggests the following trends:

Varying $\delta$ : For $N \leq N_{0}$ or, equivalently, for $\delta \leq \frac{R(\beta-1)}{N-R}$, it holds that $\frac{\vartheta P o A}{\vartheta \delta}>0$. Therefore, the $\mathrm{P} \circ \mathrm{A}$ is strictly increasing in $\delta$. For $\delta>\frac{R(\beta-1)}{N-R}$, we get $\frac{\vartheta P_{\circ} A}{\vartheta \delta}=0$. Hence, if $\delta$ exceeds $\frac{R(\beta-1)}{N-R}$, P०A is insensitive to changes of the excess cost $\delta$.

For given charging costs and on-street parking capacity, the construction of expensive parking lots in the proximity of the on-street parking area does not work effectively, when the competition is high (see Fig. 4a,b for high $N$ values and Fig. 4c,d for low $R$ values). Otherwise, under medium or low competition, there is a monotonic trend that suggests, if possible, to decrease the distance between the two options in order to increase the efficiency of the parking search process. Overall, changes in this distance and hence, the cruising cost, are meaningless for high $\delta$ values, over $\frac{R(\beta-1)}{N-R}$. In this case, when $N>N_{0}, N_{0}-R=\frac{R(\beta-1)}{\delta}$ drivers pay the extra cruising cost and end up in a parking lot together with the $N-N_{0}$ drivers that head directly for this kind of parking space. Thus, the increase of cruising cost has a double-edge effect. On the one hand, drivers are discouraged from competing so that fewer end up paying the cruising overhead. On the other hand, failing the competition for on-street parking costs more. In addition, the total number of drivers that incur the more (less) expensive parking fee is $N-R$ $(R)$, irrespective of the exact $\delta$ value. As a result, changes in $\delta$ do not affect either the total cost spent for space in the on-street or parking lot facilities, or the aggregate cruising overhead. Thus, the social cost can be decreased by locating a parking lot in the proximity of the on-street parking area so that the additional travel distance is reduced to the point of bringing the excess cost $\delta$ below $\frac{R(\beta-1)}{N-R}$.

Although low $\mathrm{P} O A$ values denote high efficiency in the parking search process, they are not always coupled with low absolute social costs. For instance, this may happen under very intense competition, 


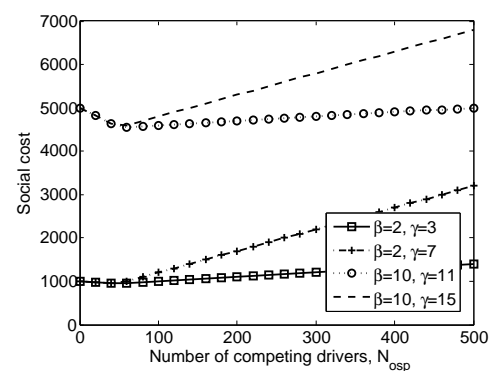

a. Pure-action profiles

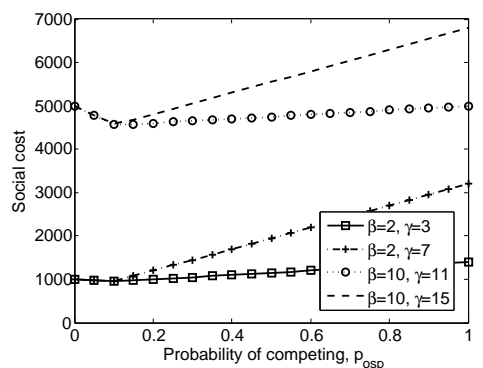

b. Symmetric mixed-action profiles

Fig. 5. Social cost for $N=500$ drivers when exactly $N_{\text {osp }}$ drivers compete (a) or when all drivers decide to compete with probability $p_{o s p}(\mathrm{~b})$, for $R=50$ on-street parking spots, under different pricing schemes.

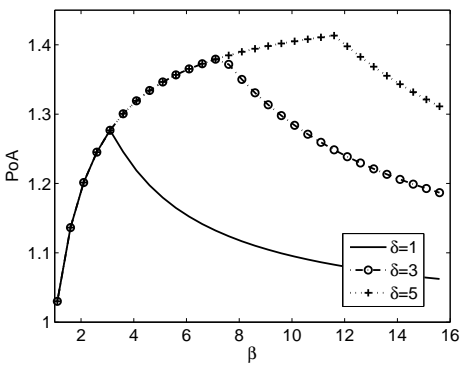

a. $2 D \mathrm{P} \circ \mathrm{A}(\beta), R=160$

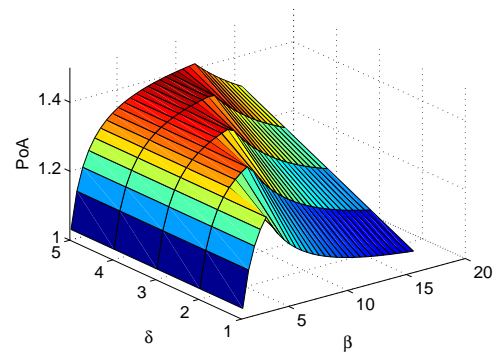

b. $3 D \mathrm{P} \circ \mathrm{A}(\beta, \delta), R=160$

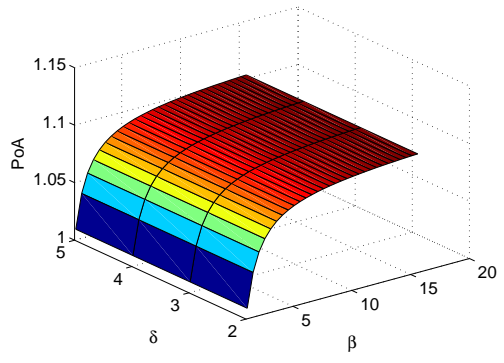

c. $3 D \mathrm{P} \circ \mathrm{A}(\beta, \delta), R=50$

Fig. 6. Price of Anarchy for $N=500$ and varying $R$, under different pricing schemes.

namely, under high parking demand (i.e., see Fig. 3 at $N=195$ ) for very low curbside capacity (i.e., see Fig. 3 at $R=10$ ). In the following section, we study the sensitivity of the social cost to the parking demand and supply as well as the prices charged for the two types of parking facilities.

\section{B. Impact of pricing scheme}

Figure 5 plots the social costs $C\left(N_{\text {osp }}\right)$ under pure (Eq. 8) and $C\left(p_{o s p}\right)$ under mixed-action strategies as a function of the number of competing drivers $N_{o s p}$ and competition probability $p_{o s p}$, respectively, where

$$
C(p)=c_{o s p, s} \sum_{n=0}^{N}\left(\begin{array}{c}
N \\
n
\end{array}\right) p^{n}(1-p)^{N-n} \cdot[\min (n, R)+\max (0, n-R) \gamma+(N-n) \beta]
$$

Figure 5 motivates two remarks. First, the social cost curves for pure and mixed-action profiles have the same shape. This comes as no surprise since for given $N$, any value for the expected number of competing players $0 \leq N_{o s p} \leq N$ can be realized through an appropriate choice of the symmetric mixed-action profile $p$. Second, the cost is minimized when the number of competing drivers is equal to the number of on-street parking spots. The cost rises when either the competition exceeds the available on-street parking capacity or the drivers are overconservative in (and refrain more than they should from) 
competing for on-street parking. In both cases, the drivers pay the penalty for the lack of coordination in their decisions. The deviation from optimal grows faster with increasing price differential between the on-street spots and the space in parking lot $($ i.e., $\beta)$ or the distance between the on-street and parking lot facilities (i.e., $\delta$ ).

If $N>R$, in the worst-case equilibrium (i.e., the equilibrium state with the maximum number of competing drivers and hence, the maximum social cost, among all equilibria) the number of drivers that actually compete for on-street parking spots exceeds the real curbside parking capacity by a factor which is a function of $\beta$ and $\gamma$ (equivalently, $\delta$ ) (see Lemma 3.1, Theorem 3.1). This inefficiency is captured in the POA plots in Figures $6 \mathrm{a}, 6 \mathrm{~b}$ for $\beta$ and $\delta$ ranging in $[1.1,16]$ and $[1,5]$, respectively. The plots illustrate the following trends:

Varying $\beta$ : For $N \leq N_{0}$ or, equivalently, for $\beta \geq \frac{\delta(N-R)+R}{R}$, it holds that $\frac{\vartheta P_{o} A}{\vartheta \beta}<0$ and therefore, the $\mathrm{P} \circ \mathrm{A}$ is strictly decreasing in $\beta$. On the contrary, for $\beta<\frac{\delta(N-R)+R}{R}$, the $\mathrm{P} \circ \mathrm{A}$ is strictly increasing in $\beta$, since $\frac{\vartheta P o A}{\vartheta \beta}>0$.

Practically, the equilibrium strategy emerging from this kind of assisted parking search behavior can approximate the optimal coordinated mechanism (i.e., POA approaches 1), provided that the operation of parking lots properly accounts for the drivers' preferences as well as estimates of the typical parking demand and supply. More specifically, if, as part of the pricing policy, the fee of parking lot is less than $\frac{\delta(N-R)+R}{R}$ times the cost of on-street parking, then the social cost in the equilibrium profile approximates the optimal social cost as the price differential between on-street and parking lot decreases. This result is inline with the statement in [12], arguing that "price differentials between on-street and off-street parking should be reduced in order to reduce traffic congestion".

Note that the $\mathrm{P} \circ \mathrm{A}$ metric also decreases monotonically for high values of the parking lot fee, specifically when the parking operator desires to gain more than $\frac{\delta(N-R)+R}{R}$ times the cost of on-street parking, towards a bound that depends on the excess $\operatorname{cost} \delta$. Nevertheless, these operating points correspond to high absolute social cost, i.e., the minimum achievable social cost is already unfavorable due to the high fee paid by the $N-R$ drivers that use space in parking lots (see Fig. 5, large $\beta$ ). However, there are instances, as in the case of $R=50$ (see Fig. 6c), where the value $\frac{\delta(N-R)+R}{R}$ corresponds to a non-realistic (too large) option for the cost of the space in parking lots, already for $\delta>1$. Thus, contrary to the previous case, the $\mathrm{P} O \mathrm{~A}$ only improves as the cost for parking lot decreases. 

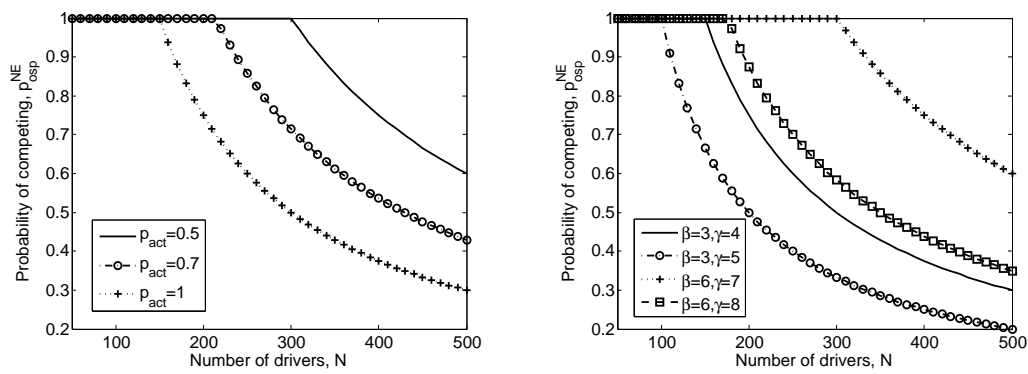

Fig. 7. Probability of competing in equilibrium, for $R=50$. Left: Strategic and Bayesian games under fixed pricing scheme $\beta=5, \gamma=7$. Right: Strategic games under various pricing schemes $\beta \in\{3,6\}, \gamma \in\{4,5,7,8\}$.

\section{Impact of information about parking demand}

Looking at the mixed-action equilibria, Theorem 3.2 indicates that drivers' intention to compete for on-street parking resources is shaped by the pricing schemes, the number of players and the curbside parking capacity. Indeed, players start to withdraw from competition as competition intensity rises over the threshold $N_{0}=\frac{R(\gamma-1)}{\delta}$. For the Bayesian implementation, the rationale behind the active players' behavior is almost the same. The only difference is that the players adjust their strategies, based on estimations for the demand level as expressed in the commonly known probabilistic information of competition. Therefore, the probability to compete decreases with the expected number of competitors $N p_{a c t}$, if this number exceeds the threshold $N_{0}$ of the strategic games (see Theorem 4.1). Furthermore, for both game formulations, players start to renege from competition as the distance between on-street and parking lot facilities (i.e., $\delta$ ) is increased or the number of opportunities for curbside parking (i.e., $R$ ) decreases or the price for space reservation in parking lot (i.e., $\beta$ ) drops. Figure 7 depicts the effect of these parameters on the equilibrium mixed-action, for strategic $\left(p_{a c t}=1\right)$ and Bayesian $\left(p_{a c t} \in\{0.5,0.7\}\right)$ games.

Less-is-more phenomena under uncertainty: Less intuitive are the game dynamics in its pre-Bayesian variant, when users only possess an estimate of the maximum number of drivers that are potentially interested in parking space. From Proposition 4.1, the mixed-action safety-level equilibrium corresponds to the mixed-action equilibrium of the strategic game $\Gamma(N)$. However, we have seen that, when the players outnumber the on-street parking capacity: a) the mixed-action equilibrium in the strategic game generates higher expected number of competitors than the optimal value $R$ (see Theorem 3.2); b) the

social cost conditionally increases with the probability of competing (see Fig. $5 \mathrm{~b}$, for $p_{\text {osp }}>\frac{R}{N}$ ); c) the probability of competition decreases with the number of players $N$ (see Fig. 7, for $N>N_{0}$ ). Therefore, at the safety-level equilibrium of the game, the drivers end up randomizing the pure action "on-street" 
with a lower probability than that corresponding to the game they actually play, with $k \leq N$ players. Hence, the resulting number of competing vehicles is smaller and, cumulatively, they may end up paying less than they would if they knew deterministically the competition they face.

One question that becomes relevant is for which (real) number $K$ of competing players do the drivers

end up paying the optimal cost. Practically, if $p_{N}^{N E}=\left(p_{o s p, N}^{N E}, p_{p l, N}^{N E}\right)$ denotes the symmetric mixed-action equilibrium for $\Gamma(N)$, we are looking for the value of $K$ satisfying:

$$
K p_{o s p, N}=R \Rightarrow K=\frac{R N}{N_{0}}=\frac{\delta}{\gamma-1} N
$$

namely, when $\frac{\delta}{\gamma-1} N$ (rounded to the nearest integer) drivers are seeking for parking space under uncertainty conditions, in the induced equilibrium they end up paying the minimum possible cost, which is better than what they would pay under complete information about the parking demand.

\section{RELATED WORK}

In several instances of ICT systems and applications a number of selfish agents compete over a limited-capacity and low-cost resource. In several studies, the way the competition is resolved in different autonomic environments has been addressed in game theoretic terms. Starting from a transportation paradigm in [13], the drivers choose between a number of routes from a common-to-all origin to a common-to-all destination. The cost incurred by each user is a non-decreasing function of the number of other users also follow the same trajectory. Likewise, in an instance of the access-point association problem, a number of mobiles compete over a finite number of access points where high access delays (low throughput) occur when many users associate with the same wireless access point [14]. In [15], nodes' access in a shared communication channel is regulated by the CSMA/CA protocol whereby the backoff rates are determined in distributed manner by self-organized nodes that either act selfishly (optimize their own throughput) or are willing to cooperate aiming at a global optimal solution. In [16], the authors present the technical challenges in design, control, and implementations of smart grids and propose game theoretic frameworks to describe the interaction-competition between the loads over the energy resources as well as between the sources over the supply of energy.

As far as the parking space-resource is concerned, the general problem of parking space search has seen contributions from different scientific disciplines such as economics, transportation, operations research, and computer science. Its first formulations appeared in the context of the broader family of stopping problems. In [17] parking spots are spread randomly with density $\lambda$ over equal-size blocks that are adjacent to the driver's travel destination. The driver circles through them, crossing the destination every 
time such a circle is over, and upon encountering a vacant spot she has to decide whether to take it or skip it and seek for a better one. Ferguson in [18] considers a simpler variant of the problem, whereby the driver's destination lies in the middle of an infinite-length straight line with parking spots that are occupied with probability $p$. In either case, the optimal policy for the drivers is shown to be of the threshold type: they should occupy an available vacant parking spot whenever this lies within some distance $r=f(\lambda)$, resp. $f(p)$, from their destination and continue searching otherwise.

On the assisted parking search front, initial work focused on centralized parking (reservation) systems. The system in [19] consists of four components: an on-board device located in the vehicle, intelligent network enabled lampposts, a sensor network that monitors the availability of parking places and a centralized parking spot scheduling/reservation server. Likewise, in the architecture in [2], every parking lot runs a reservation authority that collects parking requests via the Internet, extracts real-time statistics about its parking availability and routes them to a centralized management system that dynamically determines and broadcasts the parking fees, drawing on the relationship between parking demand and supply. Both systems are shown to better distribute the car traffic volume. Along the same line, Lu et al. in [20] propose SPARK for reducing the parking search delay. SPARK consists of three distinct services, i.e., real-time parking navigation, intelligent antitheft protection and friendly parking information dissemination, all making use of roadside network infrastructure. Finally, the authors in [1] design, implement, and evaluate a system that generates a real-time map of parking space availability. The map is constructed at a central server out of aggregate data about parking space occupancy, collected by vehicles circulating in the considered area. In a similar approach, the Parkomotivo system in [21] has been launched to monitor through a dedicate wireless sensor network and analyze using a data mining engine, on-street parking patterns in the city of Lugano (Switzerland). Drivers' can be informed by the Parkomotivo's tweet stream about the collected real-time parking availability data.

Work on opportunistic parking search assistance, where information about the location and vacancy of parking spots is opportunistically disseminated among vehicles, is rarer and more recent. In [22] the vehicle nodes solve a variant of the Time-Varying Travelling Salesman problem while dynamically planning the best feasible trip along all (reported to be) vacant parking spots. The solution attempts to minimize the total transit cost of the travelled path taking account of the time needed to reach a parking spot, the walking time from the spot to the actual destination and the probability to find the spot available. Despite the interesting treatment of the parking problem, it makes in advance the rather debatable assumption that vehicles' trips follow necessarily all reported spots. Moreover, the applied cost function may paradoxically prioritize a parking spot of lower over another of higher availability, 
when they tie in all other criteria (time to park, walking time). In [3], vehicles are allowed to exchange aggregate parking information of variable - low - accuracy in order to limit the volume of disseminated information for the sake of scalability. Simulation measurements and conclusions are derived for the profile of nodes' cache entities (i.e., information dissemination rates, freshness and spatial distribution of information in nodes' cache) under full or selective dissemination. On the other hand, the way the opportunistic exchange of information among vehicles may sharpen competition for parking space is treated in [23] and [4]. In [23], Kokolaki et al. simulate a fully cooperative opportunistic parking space assistance scheme and show that the full exchange of information upon encounters of vehicles may give rise to synchronization effects (vehicles are steered towards similar locations), sharpen competition, and eventually render the search process inefficient. Motivated by similar findings, Delot et al. propose in [4] a distributed virtual parking space reservation mechanism, whereby vehicles vacating a parking spot selectively distribute this information to their proximity. Hence, they mitigate the competition for the scarce parking spots by opportunistically controlling the diffusion of the parking information among drivers. Interestingly, the systems in [24] and [25] realize almost the same idea for parking management in the cities of Athens (Greece) and New York, respectively. In particular, both applications leverage the social network element: users can offer their parking spot to the rest of the users or find a parking spot for themselves by claiming a spot another user is offering. A rating mechanism on drivers' sharing and reserving habits, shapes parking spot seekers' likelihood to be chosen by a parking spot sharer (defender) in [24] or get informed about a vacancy prior to other seekers in [25].

Pricing and the more general economic dimensions of the parking allocation problem are analyzed from a microeconomical point of view in [26]. Anderson and de Palma view the parking spots as common property resource and question whether free access or some pricing structure result in more efficient use of the parking capacity. Working on a simple model of city and parking spot distribution, they show that this use is more efficient (in fact, optimal) when the spots are charged with the fee chosen in the monopolistically competitive equilibrium under private ownership; whereas drivers are better off when access to the parking spots is free of charge. The degree of importance of the parking pricing policy in resolving drivers' quesswork of choosing parking space, has inspired the developers of SFpark to establish and operate an advanced parking assistance system that not only collects and distributes realtime information about meter and garage parking space in San Francisco but most importantly it uses a demand-responsive pricing mechanism to match the parking availability to the emergent demand [5].

Subsequent research contributions have explicitly catered for strategic behavior and the related gametheoretic dimensions of general parking applications. In [27], the games are played among parking facility 
providers and concern the location and capacity of their parking facility as well as which pricing structure to adopt. Whereas, in the two other works, the strategic players are the drivers. In [28], which seeks to provide cues for optimal parking lot size dimensioning, the drivers decide on the arriving time at the lot, accounting for their preferred time as well as their desire to secure a space. In a work more relevant to ours, Ayala et al. in [29] define a game setting where drivers exploit (or not) information on the location of others to occupy an available parking spot at the minimum possible travelled distance, irrespective of the distance between the spot and driver's actual travel destination. The authors present distributed parking spot assignment algorithms to realize or approximate the Nash equilibrium states.

Our work also draws on game theoretic analysis and, like [28] and [29], the decision-maker(player) is the driver; and as in [23] and [4], we are particularly concerned with a broader phenomenon, evidenced in several instances of information provision within non-cooperative environments: the double-edged impact of information dissemination on the overall process efficiency, i.e., its assistance with resource/service discovery against the sharpening of competition for its usage. On the other hand, contrary to [26], [28] and [29], we explicitly discriminate between on-street parking spots and places in parking lots as two types of resources drivers have to choose among. We argue that this dilemma between searching for cheaper yet non-guaranteed on-street parking space and heading directly for the granted yet costlier parking lot(s) is a frequently recurring situation in real urban every day life; and we set our focus on the impact of information availability and accuracy on the efficiency of these decisions. Moreover, rather than considering a particular system or algorithm, as [1], [2] - [5], [19] - [22], [24] and [25] do, we leverage the game-theoretic abstractions and formulate the games corresponding to the different levels of information accuracy. This way, besides confirming pricing guidelines that have been reported in earlier work, we can derive closed-form expressions for the stable operational points in the different game settings and insightful counterintuitive results about the impact of information on the efficiency of the parking search process.

\section{CONCLUSIONS - Discussion}

In this paper, we draw our attention on fundamental determinants of parking assistance systems' efficiency rather than particular realizations. We have, thus, formulated the information-assisted parking search process as an instance of resource selection games to assess the ultimate impact that different types of parking assistance systems, collecting and sharing information of variable accuracy on parking demand, can have on the parking space selection process. Three variants of the parking resource selection game (strategic, Bayesian, and pre-Bayesian) provide normative prescriptions for the impact of information 
on drivers's decisions. In fact, this work sets some bounds on what may be achievable under rational, strategic software agents that aim at minimizing the cost of their decisions. The ultimate cost is incurred by the actual humans/drivers who are compliant with what the software agents' suggest for them. Our results dictate plausible conditions under which different charging schemes and information amounts for the parking demand, steer the equilibrium strategies, reduce the inefficiency of the parking search process, and favor the social welfare. Actually, the dissemination of parking information constitutes an instance of service provision within competitive networking environments, where more information does not necessarily improve the efficiency of service delivery but, even worse, may hamstring users' efforts to maximize their benefit. This result, obtained under the particular full rationality assumptions (i.e., software agents with perfect knowledge and free of any time and computational constraints), has direct practical implications since it challenges the need for more elaborate information mechanisms and promotes certain policies for information dissemination for the service provider.

From a methodological point of view, our expectation is that the obtained results may be deemed relevant to a broader class of service provision scenarios within competitive contexts. For instance, in a variant of the Access Point Association problem, wireless nodes compete to gain access to the lowcost (or free of charge) WLAN APs, having as a second option the more expensive service of a cellular network. Likewise, just as the tragedy-of-commons effects could increase the expected cost in the parking scenario, they could cause network blockings or degradation of the experienced service performance in this networking paradigm. Furthermore, the information $v s$. competition dynamics of the parking problem are reminiscent of those in auction-based frameworks for service provision [30] [31]. Thereby, it is the auctioneer who holds private information on the auction set-up. Announcing it to bidders, she can modulate their strategies, escalating or moderating competition and hence determining the outcome of the auction procedure, i.e., resource winners and their payments [32] [33]. Interestingly, auctions also present an alternative mechanism for charging parking facilities as opposed to the conventional two-level fixed charging scheme considered in this paper. Our first results in this direction are reported in [34].

In the remainder of this section, we iterate on two implicit assumptions behind the game models we introduced in Sections III and IV, which can motivate further research work.

Drivers' indifference among individual parking spots: The formulation of the parking spot selection game assumes that drivers do not have any preference order over the $R$ on-street parking spots. This could be the case when these $R$ spots are quite close to each other, resulting in practically similar driving times to them and walking times from them to the drivers' ultimate destinations. When drivers express preferences over different parking spots, we come up with an instance of the stable marriage problem, 
potentially with indifference [35], whereby the option of parking lot would commonly rank as the last one for all drivers. The problem objective is to derive a matching between drivers and parking spots/lots such that no subset of the drivers could be better off if they exchanged their allocated spots with each other. At a theoretical level, the search is for mechanisms that treat all drivers fairly, are strategy-proof, i.e., the drivers are motivated to advertise their true preference orders because they cannot gain by lying about them, and efficient in some Pareto-optimality sense.

Drivers' rationality: Full (or global) rationality demands an exhaustively analysis of the possible strategies available to decision-makers, identification of the equilibrium profile, and realization of the respective actions. This is more plausible when the decision-makers are automatic software agent implementations, as in our setting. The ground truth that would allow some evaluation of our results would come out of real experimentation with in-field experiments. However, there is no experimentation with such intelligent devices onboard the vehicles and the way the driver interacts with them. An alternative and subject of future work is to exploit dynamic driving emulators that are available at few selected places all over the world (e.g., the University of Massachusetts driving simulator at Amherst), to generate the appropriate software scenarios (plots) and experiment with real subjects (drivers) under close-realistic conditions.

When humans are actively involved in the decision-making process the assumption of full rationality becomes much more problematic. Indeed, Simon, already more than half a century ago [36], challenged both the normative and descriptive capacity of the fully rational decision-maker, arguing that human decisions, are most often made under knowledge, time and computational constraints. One way to accommodate the first constraints is through (pre-)Bayesian games of incomplete information; whereas the latter ones are well expressed in alternative solution concepts to the Nash Equilibrium [37] [38], arguing that "individuals are more likely to select better choices than worse choices, but do not necessarily succeed in selecting the very best choice". However, models that completely depart from the utility-maximization norm and draw on fairly simple cognitive heuristics, e.g., [39], reflect better Simon's argument that humans are satisficers rather than maximizers. For example, the authors in [40] explore the impact of the fixed-distance heuristic on a simpler version of the unassisted parking search problem. The comparison of normative and more descriptive decision-making modeling approaches both in the context of the parking spot selection problem and more general decision-making contexts, is an interesting area worth of further exploration. 


\section{REFERENCES}

[1] S. Mathur, T. Jin, N. Kasturirangan, J. Chandrasekaran, W. Xue, M. Gruteser, and W. Trappe, "Parknet: Drive-by sensing of road-side parking statistics," in Proc. 8th ACM MobiSys, San Francisco, CA, USA, 2010, pp. 123-136.

[2] H. Wang and W. He, "A reservation-based smart parking system," in Proc. 5th International Workshop on Cyber-Physical Networking Systems (colocated with IEEE INFOCOM), April 2011.

[3] M. Caliskan, D. Graupner, and M. Mauve, "Decentralized discovery of free parking places," in Proc. 3rd VANET (in conjunction with ACM MobiCom), Los Angeles, CA, USA, 2006.

[4] T. Delot, N. Cenerario, S. Ilarri, and S. Lecomte, "A cooperative reservation protocol for parking spaces in vehicular ad hoc networks," in Proc. 6th Inter. Conference on Mobile Technology, Application and Systems, ser. MOBILITY, 2009.

[5] SFpark: parking system for San Francisco, available online in http://sfpark.org/.

[6] G. Hardin, "The tradegy of the commons," Science, New Series, vol. 162, no. 3859, pp. 1243-1248, 1968.

[7] I. Ashlagi, D. Monderer, and M. Tennenholtz, "Resource selection games with unknown number of players," in Proc. AAMAS '06, Hakodate, Japan, 2006.

[8] http://www.city-parking-in-europe.eu/.

[9] A. C. Pigou, The economics of welfare. Macmillan, London, 1920.

[10] E. Koutsoupias and C. H. Papadimitriou, "Worst-case equilibria," Computer Science Review, vol. 3, no. 2, pp. 65-69, 2009.

[11] S.-G. Cheng, D. M. Reeves, Y. Vorobeychik, and M. P. Wellman, "Notes on the equilibria in symmetric games," in Proc. 6th Workshop On Game Theoretic And Decision Theoretic Agents (colocated with IEEE AAMAS), New York, USA, August 2004.

[12] R. C. Larson and K. Sasanuma, "Congestion pricing: A parking queue model," Journal of Industrial and Systems Engineering, vol. 4, no. 1, pp. 1-17, 2010.

[13] R. Holzman and N. L. yone (Lev-tov), "Network structure and strong equilibrium in route selection games," Mathematical Social Sciences, vol. 46, p. 193Ü205, 2003.

[14] C. Hasan, E. Altman, J.-M. Gorce, and M. Haddad, "Non-cooperative association of mobiles to access points revisited," in Proc. 10th Inter. Symposium on Modeling and Optimization in Mobile, Ad Hoc and Wireless Networks (WiOpt), Paderborn, Germany, 2012.

[15] Z. Shi, C. Beard, and K. Mitchell, "Competition, cooperation, and optimization in multi-hop csma networks with correlated traffic," International Journal of Next-Generation Computing, vol. 3, no. 3, 2012.

[16] W. Saad, Z. Han, H. V. Poor, and T. Basar, "Game-theoretic methods for the smart grid: An overview of microgrid systems, demand-side management, and smart grid communications," IEEE Signal Processing Magazine, 2012.

[17] J. MacQueen and J. Miller, “Optimal persistence policies,” Operations Research, vol. 8, no. 3, pp. 362-380, May-June 1960.

[18] T. S. Ferguson, "Optimal stopping and applications," online: http://www.math.ucla.edu/ tom/Stopping/.

[19] J. Boehle, L. Rothkrantz, and M. V. Wezel, “CBPRS: A city based parking and routing system,” Erasmus Research Institute of Management (ERIM), University Rotterdam, Tech. Rep. ERS-2008-029-LIS, 2008.

[20] R. Lu, X. Lin, H. Zhu, and X. Shen, "SPARK: A new VANET-based smart parking scheme for large parking lots," in Proc. IEEE INFOCOM 2009, Rio de Janeiro, Brazil, April 2009.

[21] Parkomotivo: parking system for the city of Lugano, available online in http://www.bmob-park.com.

[22] V. Verroios, V. Efstathiou, and A. Delis, "Reaching available public parking spaces in urban environments using ad hoc networking," in Proc. 12th IEEE MDM, Beijing, China, 2011. 
[23] E. Kokolaki, M. Karaliopoulos, and I. Stavrakakis, "Opportunistically assisted parking service discovery: Now it helps, now it does not," Pervasive and Mobile Computing, vol. 8, no. 2, pp. 210 - 227, 2012.

[24] Parking Defenders mobile application, available online in http://www.parkingd.com.

[25] ParkShark: parking system for New York City, available online in http://www.parkshark.mobi/www/.

[26] S. P. Anderson and A. de Palma, "The economics of pricing parking," Journal of Urban Economics, vol. 55, no. 1, pp. 1 $-20,2004$.

[27] R. Arnott, "Spatial competition between parking garages and downtown parking policy," Transport Policy (Elsevier), pp. 458-469, 2006.

[28] M. Arbatskaya, K. Mukhopadhaya, and E. Rasmusen, “The parking lot problem,” Department of Economics, Emory University, Atlanta, Tech. Rep., 2007.

[29] D. Ayala, O. Wolfson, B. Xu, B. Dasgupta, and J. Lin, "Parking slot assignment games," in Proc. 19th ACM SIGSPATIAL GIS, 2011.

[30] www.adwords.google.com.

[31] I. F. Akyildiz, W.-Y. Lee, M. C. Vuran, and S. Mohanty, "Next generation/dynamic spectrum access/cognitive radio wireless networks: A survey," Computer Network Journal (Elsevier), vol. 50, pp. 2127-2159, 2006.

[32] P. R. Milgrom and R. J. Weber, “A Theory of Auctions and Competitive Bidding,” Econometrica, pp. $1089-1122,1982$.

[33] S. Board, "Revealing information in auctions: the allocation effect," Economic Theory, pp. 125-135, 2009.

[34] E. Kokolaki, M. Karaliopoulos, and I. Stavrakakis, “Trading public parking space,” Dept. of Informatics and Telecommunications, Univ. of Athens, http://cgi.di.uoa.gr/ grad0947/parking_auctions_TR.pdf, Tech. Rep., 2012.

[35] R. W. Irving, "Stable marriage and indifference," Discrete Applied Mathematics, vol. 48, no. 3, pp. 261 - 272 , 1994.

[36] H. A. Simon, "A behavioral model of rational choice," The Quarterly Journal of Economics, vol. 69, no. 1, pp. 99-118, February 1955.

[37] R. Rosenthal, "A bounded-rationality approach to the study of noncooperative games," Int. J. Game Theory, pp. 273-292, 1989.

[38] R. McKelvey and T. Palfrey, "Quantal response equilibria for normal form games," Games and Economic Behavior, pp. 6-38, 1995.

[39] D. G. Goldstein and G. Gigerenzer, "Models of ecological rationality: The recognition heuristic," Psychological Review, vol. 109, no. 1, pp. 75-90, 2002.

[40] J. M. C. Hutchinson, C. Fanselow, and P. M. Todd, Ecological rationality: intelligence in the world. New York: Oxford University Press, 2012.

[41] D. Monderer and L. S. Shapley, "Potential games," Games and Economic Behavior, vol. 14, no. 1, pp. 124-143, May 1996.

\section{APPENDIX A}

\section{PURE EQUILIBRIA OF $\Gamma(N)$ VIA THE POTENTIAL FUNCTION}

The game $\Gamma(N)$ is a congestion game; thus, it accepts an exact potential function $\Phi(\cdot)$ [41]. As discussed in Section III, the $2^{N}$ different action profiles of $\Gamma(N)$ can be grouped into $N+1$ different meta-profiles $(m, N-m), 0 \leq m \leq N$, where $m$ is the number of drivers that decide to compete for on-street parking. Therefore, the potential function is effectively a function of $m$ and can be written as 


$$
\Phi(a) \sim \Phi(m)=\sum_{j \in \mathcal{R}} \sum_{k=0}^{n_{j}(a)} w_{j}(k)
$$

where $n_{j}(a)$ the number of drivers using resource $j$ under action profile $a$. Therefore, for $m \leq R$,

$$
\Phi(m)=(N-m) c_{p l}+\sum_{k=1}^{m} c_{o s p, s}=c_{o s p, s}[\beta N-(\beta-1) m]
$$

whereas, for $m>R$

$$
\begin{aligned}
\Phi(m) & =(N-m) c_{p l}+\sum_{k=1}^{m} \min \left(1, \frac{R}{k}\right) c_{o s p, s}+\left[1-\min \left(1, \frac{R}{k}\right)\right] c_{o s p, f} \\
& =c_{o s p, s}\left[\beta N+\delta m-R(\gamma-1)+R(1-\gamma) \cdot \sum_{k=R+1}^{m} \frac{1}{k}\right] \\
& =c_{o s p, s}\left[\beta N+\delta m-R(\gamma-1)+R(1-\gamma) \cdot\left(H_{m}-H_{R+1}\right)\right]
\end{aligned}
$$

$H_{n}=\underline{\gamma}+\log (n)+O(1 / n)$ is the $n^{\text {th }}$ harmonic number; and $\underline{\gamma}$ the Euler constant. The pure NE strategies coincide with the local minima of the potential function. For $m \leq R, \partial \Phi(m) / \partial m<0$ and the minimum is obtained at $m$, as derived in Theorem 3.1.

For $m>R$, demanding $\partial \Phi(m) / \partial m=0$ we get $\delta+\frac{R(1-\gamma)}{m_{N E}}=0$, which yields $m_{N E}=\frac{R(\gamma-1)}{\delta}=N_{0}$, i.e., the value we got through the analysis in Section III.

\section{APPENDIX B}

\section{PROOF OF THEOREM 3.2}

The symmetric equilibrium for $N \leq N_{0}$ corresponds to the pure NE we derived in Theorem 3.1. To compute the equilibrium for $N>N_{0}$ we invoke the condition that equilibrium profiles must fulfil

$$
c_{i}^{N}\left(o s p, p^{N E}\right)=c_{i}^{N}\left(p l, p^{N E}\right)
$$

namely, the costs of each pure action belonging to the support of the equilibrium mixed-action strategy are equal. Hence, from (10) and (15) the symmetric mixed-action equilibrium $p^{N E}=\left(p_{o s p}^{N E}, p_{p l}^{N E}\right)$ solves the equation

$$
f(p)=-\beta+\sum_{k=0}^{N-1}\left(\gamma-\min \left(1, \frac{R}{k+1}\right) \cdot(\gamma-1)\right) B(k ; N-1, p)=0
$$

A closed-form expression for the equilibrium $p_{o s p}^{N E}$ is not straightforward. However, it holds that:

$$
\lim _{p \rightarrow 0} f(p)=-\beta+1<0 \text { and } \lim _{p \rightarrow 1} f(p)=\delta\left(1-\frac{N_{0}}{N}\right)>0
$$


and $f(p)$ is a continuous and strictly increasing function in $p$ since

$$
f^{\prime}(p)=\sum_{k=0}^{N-1}\left(\gamma-\min \left(1, \frac{R}{k+1}\right)(\gamma-1)\right) B^{\prime}(k ; N-1, p)>\sum_{k=0}^{N-1} B^{\prime}(k ; N-1, p)=0
$$

Hence, $f(p)$ has a single solution. It may be checked with replacement that $f\left(N_{0} / N\right)=0$.

\section{APPENDIX C}

\section{PROOF OF THEOREM 4.1}

Inline with the reasoning in the proof of Theorem 3.2, any symmetric mixed-action equilibrium $p^{N E_{B}}$ must fulfill

$$
c_{i}^{N_{B}}\left(o s p, p^{N E_{B}}\right)=c_{i}^{N_{B}}\left(p l, p^{N E_{B}}\right)
$$

Since $c_{i}^{N_{B}}(p l, p)=c_{p l}$ and $c_{i}^{N_{B}}(o s p, p)=\sum_{n_{a c t}=0}^{N-1} c_{i}^{n_{a c t}+1}(o s p, p) B\left(n_{a c t} ; N-1, p_{a c t}\right)$, a few algebraic manipulations suffice to derive that the symmetric mixed-action equilibrium $p^{N E_{B}}$ solves the equation

$$
h(p)=-\beta+\sum_{n_{a c t}=0}^{N-1} B\left(n_{\text {act }} ; N-1, p_{\text {act }}\right) \cdot \sum_{k=0}^{n_{\text {act }}}\left(\gamma-\min \left(\frac{R}{k+1}, 1\right) \cdot(\gamma-1)\right) B\left(k ; n_{\text {act }}, p\right)=0
$$

The function $h(p)$ is continuous and strictly increasing in $p$ for all $p_{a c t} \in[0,1]$ since

$$
\begin{aligned}
h^{\prime}(p) & =\sum_{n_{a c t}=0}^{N-1} B\left(n_{a c t} ; N-1, p_{a c t}\right) \cdot \sum_{k=0}^{n_{a c t}}\left(\gamma-\min \left(\frac{R}{k+1}, 1\right) \cdot(\gamma-1)\right) B^{\prime}\left(k ; n_{a c t}, p\right) \\
& >\sum_{n_{a c t}=0}^{N-1} B\left(n_{a c t} ; N-1, p_{a c t}\right) \sum_{k=0}^{n_{a c t}} B^{\prime}\left(k ; n_{a c t}, p\right) \\
& =\sum_{n_{a c t}=0}^{N-1} B\left(n_{a c t} ; N-1, p_{a c t}\right)\left(\sum_{k=0}^{n_{a c t}} B\left(k ; n_{a c t}, p\right)\right)^{\prime}=0
\end{aligned}
$$

since the weights of the rightmost Binomial coefficients in the second line are not smaller than one,

$$
\gamma-\min \left(\frac{R}{k+1}, 1\right) \cdot(\gamma-1)>1, \forall k \in[0, N-1]
$$

Likewise, for $p_{\text {act }} \in[0,1]$, its leftmost value is $\lim _{p \rightarrow 0} h(p)=-\beta+1<0$ and its rightmost value is

$$
\lim _{p \rightarrow 1} h(p)=-\beta+\sum_{n_{\text {act }}=0}^{N-1} B\left(n_{\text {act }} ; N-1, p_{\text {act }}\right)\left(\gamma-\min \left(1, \frac{R}{n_{\text {act }}+1}\right) \cdot(\gamma-1)\right)=f\left(p_{\text {act }}\right)
$$

In the proof of Theorem 3.2 we showed that the function $f(p)$ is strictly increasing in $p$ and has a single solution $p=N_{0} / N$. Therefore, as long as $p_{a c t} \in\left[0, N_{0} / N\right), \lim _{p \rightarrow 1} h(p)<0$, and $c_{i}^{N_{B}}(o s p, p)<$ $c_{i}^{N_{B}}(p l, p) \forall p \in(0,1)$; namely, it is a dominant strategy for all drivers to compete for on-street parking. On the contrary, for $p_{a c t} \in\left[N_{0} / N, 1\right], \lim _{p \rightarrow 1} h(p)$ gets positive values and $h(p)=0$ has a single solution $p=\frac{N_{0}}{N p_{a c t}}$ (can be checked with replacement). 